\title{
Radio lobe dynamics and the environment of microquasars
}

\author{
S. Heinz ${ }^{\star}$ \\ Max-Planck-Institut für Astrophysik, 85741 Garching, Germany
}

Received 19 February 2002 / Accepted 14 March 2002

\begin{abstract}
We argue that, when compared to AGNs in dynamical terms, microquasars are found in low density, low pressure environments. Using a simple analytic model, we discuss radio lobe dynamics and emission. Dynamical considerations for GRS 1915+105 and GRO J1655-40 show that they are located in regions much less dense than the canonical $n_{\mathrm{ISM}} \sim 1 \mathrm{~cm}^{-3}$ unless the jets are unusually narrow or much more powerful than currently believed.
\end{abstract}

Key words. galaxies: jets - ISM: jets and outflows - stars: individual: GRS 1915+105, GRO J1655-40

\section{Introduction}

From the time of discovery of relativistic jets in Galactic $\mathrm{X}$-ray binaries, their morphological and physical similarity with AGN jets has been stressed in the literature (see Mirabel \& Rodríguez 1999 for a review on the subject), which has inspired qualitative comparisons of Galactic and extragalactic jets. Such comparisons are a powerful tool to study the dependence of jets on the input conditions, which is crucial for understanding the process of jet formation. While the central black holes in AGNs span 3 orders of magnitude in mass $M$, with measurements of $M$ often hampered by the lack of accurate indicators, Galactic compact objects fall into a narrow range in $M$, while extending the mass scale to a range of over 9 orders of magnitude.

So far, the comparison has focused mainly on the emission from the inner jet, while large scale (lobe) emission has traditionally been difficult to observe in Galactic jets and has thus not been considered much in the literature. This Letter presents arguments for the scaling expected on large scales. Section 2 reviews the scaling relations of jet parameters. In Sect. 3 we argue that, typically, microquasar jets are located in low density environments compared to AGN jets, derive simple scaling relations for radio lobes and put limits on the density of the surroundings of GRS 1915+105 and GRO J1655-40, Sect. 4 summarizes.

\section{Scaling of jet sources}

The conditions in the inner disk around black holes are essentially determined by three parameters: black hole mass $M$, spin $a$, and accretion rate $\dot{m}=\dot{M} / \dot{M}_{\mathrm{Edd}}\left(\dot{M}_{\mathrm{Edd}}\right.$ is the Eddington rate). Jet formation seems to be strongly dependent on $\dot{m}$, with jet activity associated with a given range in $\dot{m}$, while the influence of $a$ is still unclear. As we

* e-mail: heinzs@mpa-garching.mpg.de are interested in powerful jets, we assume that $a$ and $\dot{m}$ take on their optimal value for jet formation and consider only variations in $M$. Then, the kinetic jet luminosity $L$ follows $L \propto M \psi(\dot{m}, a)$, with some unknown function $\psi$.

Physical quantities in the inner disk take on the following scaling: size and time scales relate linearly to the fundamental length, the gravitational radius $r_{\mathrm{g}}$ of the black hole, $r \propto t \propto r_{\mathrm{g}} \propto M$. It is convenient to define natural units $\varpi \equiv r / r_{\mathrm{g}}$ and $T \equiv c t / r_{\mathrm{g}}$. It follows from dimensional arguments and standard accretion disk theory (Shakura \& Sunyaev 1976) that density and pressure scale inversely with $M: n_{\text {disk }} \propto p_{\text {disk }} \propto M^{-1}$. The magnetic pressure generated or transported in the disk will be some fraction $\varphi$ of the gas pressure: $p_{\mathrm{B}}=\varphi p_{\text {gas }} \propto M^{-1}$, thus $B \propto M^{-1 / 2}$; $\varphi$ is arbitrary but should not depend on $M$. Since jets originate in the inner disk, conditions in the inner jet should assume the same scaling: the jet cross section $R_{0}$ at injection scales with $M, R_{0} \propto M$, density and pressure scale like $M^{-1}$, and the jet power follows $L_{\text {kin }} \propto R^{2} B^{2} \propto M$.

However, not all aspects of relativistic jets take on such a simple $M$-dependence, which can be seen from the observed non-linear scaling of the radio flux of the inner jet with $M$ that has been explained successfully using only the above scaling relations and simple assumptions about jet geometry (Falcke \& Biermann 1996).

In this Letter we consider the large scale structure of jets, where interaction with the environment is important. The scaling based only on conditions in the inner disk will not hold, since parameters independent from the inner disk enter: external density $\rho_{\mathrm{x}}$ and pressure $p_{\mathrm{x}}$. Since ISM densities are typically larger than IGM densities (with the canonical ISM value of $n_{\text {ISM }} \sim 1 \mathrm{~cm}^{-3}$ ), one might think that, compared to AGN jets, Galactic jets are situated in high density environments. In the following section we will argue that this is a misconception and that the selfsimilarity in $M$ is broken on large scales. 


\section{Large scale evolution of radio sources}

\subsection{Radio lobe dynamics}

The large scale dynamics of an active source (i.e., still driven by active jets) are governed by the dimensionless ratio $\eta_{\rho} \equiv\left(\mathcal{L} / R^{2} c^{3} \rho_{\mathrm{x}}\right)$, where $R$ is the characteristic size scale (i.e., cross section) of the jet, and $\mathcal{L}$ is the mean kinetic luminosity ${ }^{1}$. Since typical dimensions of the jet are set by the inner disk and should follow $R \propto M$ (see Sect. 2), and since $\mathcal{L} \propto M$, the problem becomes scale invariant (i.e., the value of $\eta_{\rho}$ independent of $M$ ) if $\rho_{\mathrm{x}} \propto M^{-1}$

IGM densities fall into the range $10^{-5} \mathrm{~cm}^{-3} \lesssim n_{\text {IGM }} \lesssim$ $10^{-2} \mathrm{~cm}^{-3}$, while Galactic ISM densities span the range of few $\times 10^{-3} \mathrm{~cm}^{-3} \lesssim n_{\text {ISM }} \lesssim 10^{4} \mathrm{~cm}^{4}$ (lower limit: hot ISM, upper limit: molecular clouds). Thus, the similarity condition $\rho_{\mathrm{x}} \propto M^{-1}$ could only be satisfied for microquasars situated in molecular clouds. Most Galactic jets are, however, located in much lower ISM densities. Thus, compared to radio galaxies, microquasars are situated in low density environments in a dynamical sense.

A similar argument applies to the external pressure: the terminal size $\varpi_{\mathrm{t}}$ of an inactive radio lobe in natural units, when it has reached pressure equilibrium with the ISM, follows $\varpi_{\mathrm{t}} \sim\left(M p_{\mathrm{x}}\right)^{-1 / 3}$. The dynamical time in natural units will be $T \propto \varpi_{\mathrm{t}} / c_{\mathrm{sx}} \propto\left(M^{2} \rho_{\mathrm{x}} p_{\mathrm{x}}\right)^{-1 / 2}$, where $c_{\mathrm{S}, \mathrm{x}}$ is the external sound speed. Scale invariance in $M$ (i.e., quantities expressed in natural units are independent of $M$ ) would require $p_{\mathrm{x}} \propto M^{-1}$. Since IGM and ISM pressures are comparable, microquasars are, in effect, located in low pressure environments, relative to AGNs, and the equilibrium size and dynamical time scales are much larger than in AGNs when expressed in natural units.

Based on this premise, the dynamics of microquasar lobes might be qualitatively different from AGN lobes. Because observations of microquasar radio lobes are only just beginning to appear (partly due to their low brightness), it is unclear how to describe the dynamics of these sources. Numerical simulations and more radio observations are therefore necessary. Meanwhile, we can use the existing framework of AGN radio lobes for simple estimates. In turn, observations of microquasars can be used to study the lobe evolution in low density environments.

Once it has passed through the terminal shock, the spent jet fuel is deposited in the vicinity of the jet head, inflating the radio lobes. During the early (i.e., active) stage, the lobes expand supersonically into the environ-

1 The evolution time scales for AGN lobes are of order $t \sim 10^{7}-10^{8}$ yrs. Variation in $L_{\text {kin }}$ on much shorter time scales will average out, making the large scale evolution dependent only on the mean kinetic power $\mathcal{L} \equiv\left\langle L_{\text {kin }}\right\rangle$. Though the relation between $\mathcal{L}$ and $M$ is not known (possibly depending on details like binary accretion) the giant flare duration of order days observed in microquasars is only one or two orders of magnitudes shorter than the estimated life times of extragalactic jets when scaled by mass (of order $10^{6}-10^{8} \mathrm{yrs}$ ) and we will assume that the duty cycles and typical time scales of jet activity scale roughly linearly with $M$, and thus $\mathcal{L} \propto M$. ment. Later they come into pressure equilibrium. For a supersonic bubble expanding into a medium with a radial powerlaw density profile $\rho_{\mathrm{x}} \equiv \rho_{\mathrm{x}, 0}\left(r / r_{\mathrm{x}}\right)^{-\zeta}$, there exists a well known self-similar solution (Castor et al. 1975; Falle 1991) for the cocoon radius $r_{\mathrm{c}}$ :

$r_{\mathrm{c}}=A\left(\frac{\mathcal{L} t^{3}}{\rho_{\mathrm{x}}(r)}\right)^{1 / 5}=A\left(\frac{\mathcal{L} t^{3}}{\rho_{\mathrm{x}, 0} r_{\mathrm{x}}^{\zeta}}\right)^{1 /(5-\zeta)}$

with $\left.A \equiv\left[(5-\zeta)^{3}\left[36 \pi(8-\zeta)^{\frac{1+\zeta}{3}}(11-\zeta)^{\frac{2-\zeta}{3}}\right)\right]^{-1}\right]^{\frac{1}{5-\zeta}}$ of order unity. This scaling is still appropriate if the cocoon is not spherical and entirely sufficient for our purpose.

The solution in Eq. (1) is Rayleigh-Taylor unstable for $\zeta \geq 2$. However, the environments of AGNs and microquasars are benign: microquasars are typically located in homogeneous media $(\zeta \sim 0)$, while AGNs are typically located in stratified atmospheres with roughly uniform densities close in and steeper decline further out $(\zeta \sim 1.5)$.

If the nuclear source turns off before the lobes reach pressure equilibrium with their surroundings, a Sedov phase similar to a regular blast wave will follow, though the lobe gas will be relativistic, thus expansion will only be supersonic with respect to the external gas. If the source sits in a stratified atmosphere the lobes will rise buoyantly and cool adiabatically, once the expansion becomes subsonic (i.e., in pressure equilibrium with the environment).

\subsection{Scaling relations for emission from radio lobes}

Using Eq. (1) one can estimate the emission from the radio lobes. For a powerlaw distribution $f(\gamma)=C \gamma^{-s}$ with spectral index $s \sim 2$ this gives (e.g., Jarvis et al. 2001):

$L_{\nu} \propto \rho_{\mathrm{X}}^{\frac{3+3 s}{4(5-\zeta)}} r_{\mathrm{X}}^{\frac{\zeta(3+3 s)}{4(5-\zeta)}} \mathcal{L}^{\frac{12+(5+s)(2-\zeta)}{4(5-\zeta)}} t^{\frac{36-(5+s)(4+\zeta)}{4(5-\zeta)}}$

Using Eq. (2) we can determine the scaling of radio luminosity with the fundamental source parameters. For active sources with the same absolute age $t$, the radio flux will scale like $F_{\nu} \propto \mathcal{L}^{1.3} \rho_{\mathrm{x}}(r)^{0.45} t^{0.4} \propto M^{1.3} \rho_{\mathrm{x}}(r)^{0.45}$. Typically, however, one would expect the jet activity time scale to be proportional to the disk time scales, i.e., proportional to $M$. Comparing sources of the same scaled age $T \propto t / M$ gives $F_{\nu} \propto \mathcal{L}^{1.3} \rho_{\mathrm{x}}^{0.45} M^{0.4} T^{0.4} \propto M^{1.7} \rho_{\mathrm{x}}(r)^{0.45}$.

Thus, for sources located in uniform environments, the scaling index $\xi \equiv \mathrm{d} \ln F_{\nu} / \mathrm{d} \ln M$ will fall into the range $1.3 \leq \xi \leq 1.7$, interestingly close to the scaling measured in AGNs (e.g., Lacy et al. 2001). This limit should be valid for Galactic sources and for extragalactic sources which are still confined to the core of the cluster potential (where $\zeta \sim 0)$. In stratified atmospheres (i.e., for large AGN jets), the dependence of $F_{\nu}$ on $\rho_{\mathrm{x}}$ can lead to a much weaker $M$ dependence: for the canonical value of $\zeta \sim 1.5$, found in typical isothermal cluster atmospheres, Jarvis et al. (2001) find $\xi \sim 1.1$ for sources of the same age $t$, and for sources of the same scaled age $\xi \sim 0.9$. Finally, a useful (since measurable) comparison is for sources of the same absolute size $r_{\mathrm{c}}$, where $\xi=(5+s) / 6$, independent of $\zeta$. 


\subsection{Microquasar radio lobes}

Strictly speaking, Eq. (1) is valid only for lobes expanding at sub-relativistic speeds. Since, as argued above, microquasars are located in under-dense environments, their expansion stays relativistic much longer, measured in natural units $T \propto t / M$, which complicates the dynamics significantly, partly because the lobes are no longer in causal contact (which was the tacit assumption in deriving Eq. (1)). An analytic treatment of the evolution of relativistic lobes is beyond the scope of this Letter. Instead, we simply note that the following discussion applies only to microquasar lobes old enough to have become sub-relativistic. For the moderate Lorentz factors of $\Gamma_{\text {jet }} \sim 5$ involved, relativistic corrections should, in any case, stay within an order of magnitude, sufficient for our purposes.

We can then use Eq. (2), taking a fiducial kinetic jet luminosity of $\mathcal{L} \equiv 10^{39} \mathcal{L}_{39} \mathrm{ergs} \mathrm{s}^{-1}$ (a reasonable estimate during powerful flares like those observed in GRS $1915+105$ or GRO J1655-40) and a constant external density with $\zeta=0$ to arrive at an estimate of the absolute flux from a microquasar at distance $D=10 D_{10} \mathrm{kpc}$ of

$$
F_{\nu} \sim 100 \mathrm{mJy} n_{\mathrm{ISM}}^{0.45} \mathcal{L}_{39}^{1.3} t^{0.4}\left(\frac{2 \varphi^{3 / 4}}{1+\varphi}\right) D_{10}^{-2} \nu_{5}^{-1 / 2}
$$

where $\nu_{5}$ is the observing frequency in units of $5 \mathrm{GHz}$.

Since the lobe expansion will be supersonic for much longer than the expected lifetime of the nuclear jet, a Sedov phase will follow the active expansion phase, during which the lobe radius will roughly follow $R \propto t^{2 / 5}$ and the luminosity will follow $L_{\nu} \propto t^{-0.9}$. This phase will begin after the source switches off, at $t_{\mathrm{s}}=10^{5} \mathrm{~s} E_{44} / \mathcal{L}_{39} \approx 1$ day, and it will last until the source reaches pressure equilibrium with the surrounding medium at $t_{\mathrm{p}}$.

Since the luminosity during the Sedov phase is declining, the source flux reaches a maximum at the beginning of the Sedov phase and will then follow

$$
F_{\nu} \sim 10 \mathrm{Jy} \frac{n_{\mathrm{ISM}}^{0.45} \mathcal{L}_{39}^{0.9} E_{44}^{0.4}}{D_{10}^{2}}\left(\frac{2 \varphi^{3 / 4}}{1+\varphi}\right)\left(\frac{t}{t_{\mathrm{s}}}\right)^{-0.9} \nu_{5}^{-1 / 2}
$$

with a timescale of $t_{\mathrm{s}} \approx 1$ day. For ISM densities appropriate for the hot phase, the flux should be rather dim to begin with and fade quickly beyond detectability.

For an external pressure of $p_{\mathrm{x}} \equiv 10^{-11} \mathrm{ergs} \mathrm{cm}^{-3} p_{-11}$, the lobe reaches pressure equilibrium with the ISM when it has reached a size of $r_{\mathrm{e}} \sim 0.2 \mathrm{pc} E_{44}^{1 / 3} p_{-11}^{-1 / 3}$ on a timescale of order $t_{\mathrm{e}} \sim 4 \times 10^{3} \mathrm{yrs} E_{44}^{1 / 3} n_{\mathrm{x}}^{1 / 2} p_{-11}^{-5 / 6}$. The radio flux is then $F_{\nu} \lesssim 20 \mu \mathrm{Jy} E_{44} p_{-11}^{3 / 4} D_{10}^{-2} \nu_{5}^{-1 / 2}$ (the upper limit is set by equipartition), with surface brightness $I_{\nu} \lesssim 2 \mathrm{mJy} \mathrm{arcmin}^{-2} E_{44}^{1 / 3} p_{-11}^{17 / 12} \nu_{5}^{-1 / 2}$, i.e., a brightness temperature of $T_{\mathrm{B}} \lesssim 3 \times 10^{-2} \mathrm{~K} p_{-11}^{17 / 12} E_{44}^{1 / 3} \nu_{5}^{-5 / 2}$.

Multiple ejection events will lead to the accumulation of a faint radio halo around the source, with the value for $E_{44}$ now reflecting the total accumulated energy in the halo in the expressions for $F_{\nu}$ and $I_{\nu}$. Strong radiative losses in the early Sedov phase (during which no further injection of relativistic particles by the jet occurs) would limit the detectability of this halo to very low frequencies.

The lack of strong radio emission from lobes following the powerful outbursts from microquasars like GRS $1915+105$ and GRO J1655-40 indicates that the surrounding density should be lower than the canonical value of $1 \mathrm{~cm}^{-3}$ (see Eq. (4) and Sect. 3.4). The detection of lobe emission from Sco X-1 (at $D_{10} \sim 0.32$ ) at the $\sim 10 \mathrm{mJy}$ level and on timescales of order days (Fomalont et al. 2001) is consistent with originating from the early Sedov phase.

Persistent radio structures on pc scales have been found in the sources 1E 1740.7-2942 (Mirabel \& Rodríguez 1999) and GRS 1758-258 (Martí et al. 1998). The lack of current jet activity, compared to the relatively strong emission from the extended structure on $0.1-1 \mathrm{mJy}$ levels would argue for an epoch of powerful jet activity in the recent past. Their flux and surface brightness indicate that they are either located in overpressured regions (which would be compatible with a position close to the Galactic center) and/or that the emitting plasma has not yet reached pressure equilibrium with the ISM.

If the bright $(\sim 200 \mathrm{mJy})$ emission on arcmin scales around Cir X-1 (Haynes et al. 1986; Stewart et al. 1993) is due to past jet activity then the total energy in the emitting plasma exceeds $E_{44} \gtrsim 10^{4}$. The dynamical source age of $\lesssim 10^{5}$ yrs (Eq. (3)) agrees well with the estimated age of the associated supernova remnant SNR G321.9-0.3 (Haynes et al. 1986). This would imply onoing jet activity at an average power of $\mathcal{L}_{39} \gtrsim 10^{-4}$. Clearly, further work is required to understand each of these sources in detail.

\subsection{The environment of GRS 1915 and GRO J1655}

We shall now demonstrate that the best studied microquasars, GRS 1915+105 and GRO J1655-40, are indeed locate in low density gas. For the sake of simplicity we assume that the bright knots in the jet of GRS 1915+105 are discrete ejections. Kaiser et al. (2000) have demonstrated how the physics of the jet changes if the knots are internal shocks rather than blobs. However, as long as we consider only the total energy $E$ contained in the outflow, the internal jet structure is irrelevant for this argument.

VLBI and MERLIN observations of the 1994 and 1997 events (Mirabel \& Rodríguez 1994; Fender et al. 1999) show that the knots traveled out to a distance of at least $0.04 \mathrm{pc}$ (set by the detection limit, i.e., the knots might well have traveled further). The observed velocity of the components is constant out to at least this distance.

The length of the jet is already an indication that the interaction with the ISM must be much less efficient in this jet than it is in extragalactic objects: when scaled by central mass, the length of $l \gtrsim 0.04$ pc observed in GRS 1915+105 would translate to a jet length of $l \gtrsim 4 \mathrm{Mpc}$ for a jet with a supermassive black hole at its center, like M87 or Cyg A. This is longer than any observed AGN jets - even in giant radio galaxies like 
NGC 315 (Bridle et al. 1976), and this is only a lower limit to the jet length!

Based on equipartition arguments, the kinetic energy in the ejection is roughly $E_{\text {kin }} \sim 10^{44} E_{44}$ ergs (Fender et al. 1999; Rodríguez \& Mirabel 1999). As it travels downstream, we assume that the knot expands conically with an opening angle $\theta \equiv 5^{\circ} \theta_{5}$ (i.e., a half-opening angle of $2.5^{\circ}$ ), and sweeps up (or pushes aside) the ambient matter in its way (e.g., Heinz \& Begelman 1999). The ejection will have been slowed down by the interaction with the external matter once it has swept up a fraction of $1 / \Gamma$ of its own mass. Thus, for an external particle density of $n_{\mathrm{x}}$, the ejection will slow down at a distance

$d_{\text {slow }} \sim 10^{16} \mathrm{~cm}\left(E_{44} / \Gamma_{5}^{2} n_{\mathrm{x}} \theta_{5}^{2}\right)^{1 / 3}$,

where $\Gamma_{5} \equiv \Gamma / 5$ is the Lorentz factor of the jet.

Comparing this with the observed distance of the ejections, $d_{\text {obs }} \gtrsim 1.3 \times 10^{17} \mathrm{~cm} D_{11} \sin \left(66^{\circ}\right) / \sin \left(\vartheta_{\text {LOS }}\right)$ (where $D_{11}$ is the distance to GRS $1915+105$ in units of $11 \mathrm{kpc}$ and $\vartheta_{\text {LOS }}$ the viewing angle of the jet), we arrive at the following upper limit on the external density:

$n_{\mathrm{x}} \lesssim 10^{-3} \mathrm{~cm}^{-3} \frac{E_{44} \sin \left(\vartheta_{\mathrm{LOS}}\right)^{3}}{\Gamma_{5}^{2} \theta_{5}^{2} D_{11}^{3} \sin \left(66^{\circ}\right)^{3}}$.

A similar analysis can be made for the GRO J1655-40 jet, which has also been observed out to a distance of $\sim 0.04 \mathrm{pc}$ (Hjellming \& Rupen 1995), giving the same limit. Thus, these jets must be located in environments much less dense than the canonical $n_{\mathrm{ISM}} \sim 1 \mathrm{~cm}^{-3}$, unless they are very narrow $\left(\theta \lesssim 0.15^{\circ}\right)$ or very energetic, $\left(E \gtrsim 10^{47} \mathrm{ergs}\right)$.

The simplest interpretation of this result is either $(a)$ that GRS 1915+105 and GRO J1655-40 are located in a region occupied by the hot ISM phase, or $(b)$ that previous activity of the jets has created an evacuated bubble around them (i.e., the plasma halo mentioned in Sect. 3.3), filled with relativistic plasma (in GRO J1655-40, which is a HMXB, the companion could also produce such a bubble via an outflow, while in the LMXB GRS $1915+105$, a stellar origin of such a bubble would have to be attributed to the stellar wind or SN explosion of the progenitor). Since the energy requirements on such a bubble would only be of order $10^{42} \operatorname{ergs} p_{-11}$, this is energetically easily possible.

The limit set in Eq. (6) can only be avoided if the jet were traveling down an evacuated channel, pre-existing to the outburst. The stability of such a channel (without strong jet activity keeping it open, which would be observable) inside a medium much more dense than the above limits is questionable, given that there should be precession and significant proper motion between outbursts.

A very narrow opening angle would imply that the jet material is very cold or very well confined. However, the pressure of the synchrotron emitting electrons alone is already much larger than typical ISM pressures, and an external confinement is therefore excluded. Thus, a very narrow opening angle would imply that the jet material is cold. This, in turn, would increase the energy requirements on the jet (Fender et al. 1999). Energies much larger than
$10^{44} \mathrm{ergs}$, on the other hand, would require the kinetic luminosity of the central engine to severely exceed the Eddington limit $L_{\text {Edd }} \sim 10^{39} \mathrm{ergs} \mathrm{s}^{-1}$.

However, even if we allow for conservative lower limits on $\theta_{5}$ and generous energy estimates, Eq. (6) still shows that the density of the environment around the two best studies jets, GRS $1915+105$ and GRO J1655-40, is much smaller than typical molecular cloud densities, which would be needed for scale invariance with typical extragalactic objects. These estimates show that measurements of the external density and the stopping distance of the jets could be used to constrain important parameters of the jet, such as the energy and the opening angle.

\section{Conclusions}

We have demonstrated that microquasars are typically located in much less dense environments than AGN jets in a dynamical sense, resulting in a reduction of the emission from extended radio lobes, consistent with the rarity of such structures in powerful Galactic jet sources. It also explains the observed length of the jets in GRS $1915+105$ and GRO J1655-40, $l \gtrsim 0.04 \mathrm{pc}$, which would correspond to a jet length of $4 \mathrm{Mpc}$ when scaled by $M$ to AGN conditions. Estimates of the environmental density of these sources based indicate that they are located in environments much less dense than the canonical $1 \mathrm{~cm}^{-1}$, unless the jets are very narrow or extremely energetic.

Acknowledgements. I would like to thank E. Chirazov, T. Enßlin, R. Sunyaev, and the referee M. Lacy for their comments.

\section{References}

Bridle, A. H., Davis, M. M., Meloy, D. A., et al. 1976, Nature, 262, 179

Castor, J., McCray, R., \& Weaver, R. 1975, ApJ, 200, L107

Falcke, H., \& Biermann, P. L. 1996, A\&A, 308, 321

Falle, S. A. E. G. 1991, MNRAS, 250, 581

Fender, R. P., Garrington, S. T., McKay, D. J., et al. 1999, MNRAS, 304, 865

Fomalont, E. B., Geldzahler, B. J., \& Bradshaw, C. F. 2001, ApJ, 553, L27

Haynes, R. F., Komesaroff, M. M., Jauncey, D. L., et al. 1986, Nature, 324, 233

Heinz, S., \& Begelman, M. C. 1999, ApJ, 527, L35

Hjellming, R. M., \& Rupen, M. P. 1995, Nature, 375, 464

Jarvis, M. J., Rawlings, S., Lacy, M., et al. 2001, MNRAS, 326, 1563

Kaiser, C. R., Sunyaev, R., \& Spruit, H. C. 2000, A\&A, 356, 975

Lacy, M., Laurent-Muehleisen, S. A., Ridgway, S. E., Becker, R. H., \& White, R. L. 2001, ApJ, 551, L17

Martí, J., Mereghetti, S., Chaty, S., et al. 1998, A\&A, 338, L95

Mirabel, I. F., \& Rodríguez, L. F. 1994, Nature, 371, 46

Mirabel, I. F., \& Rodríguez, L. F. 1999, ARA\&A, 37, 409

Rodríguez, L. F., \& Mirabel, I. F. 1999, ApJ, 511, 398

Shakura, N. I., \& Sunyaev, R. A. 1976, MNRAS, 175, 613

Stewart, R. T., Caswell, J. L., Haynes, R. F., \& Nelson, G. J. 1993, MNRAS, 261, 593 